\title{
PET imaging of garbage protein in Alzheimer's disease: does it require reappraisal of brain PET analysis?
}

\author{
Serge Goldman
}

Published online: 11 March 2014

(C) Springer-Verlag Berlin Heidelberg 2014

At the recent Alzheimer's Disease Summit: The Path to 2025, held in November 2013, the societal and economic threat posed by Alzheimer's disease (AD) to our civilization was acknowledged. By 2050 the condition will affect 135 million people worldwide, and it will incur an annual cost of $\$ 1$ trillion, just in the US. To win the fight against the disease, the summit identified the investment in new biomarkers of the disease as a priority. A recent article by Villemagne et al. in the European Journal of Nuclear Medicine and Molecular Imaging is in line with this prioritization [1]. The article describes initial results obtained with a new marker of tau protein deposits in the brain, ${ }^{18} \mathrm{~F}-\mathrm{THK} 523$. Tau is involved in a pathophysiological process considered pivotal in Alzheimer's disease and other dementing conditions grouped under the tauopathy denomination. The development of tracers targeting tau deposition is at the forefront in the search for valid biomarkers of dementing conditions. This research field was opened with the invention of the "PiB compound", the PET tracer of beta-amyloid (Aß) deposits [2]. The various works now dedicated to tau protein detection raise a basic question: why do we need a marker of tau protein deposits since we now have access to multiple $A B$ tracers which are reported to be supreme diagnostic tools in AD? One reason is that $A B$ accumulation seems less directly related to neuronal death than the tau metabolic disturbances leading to the welldefined neurofibrillary degeneration. Also, the early enthusiasm generated by the $A B$ tracers has been attenuated by the remembrance of some basic facts established several decades ago. Among these facts are the well-established presence of $\mathrm{A} ß$ plaques in the brain of subjects in good cognitive health

S. Goldman $(\bowtie)$

PET/Biomedical Cyclotron Unit - Department of Nuclear Medicine, ULB-Hôpital Erasme, and Center for Microscopy and Molecular Imaging (CMMI), Université Libre de Bruxelles, 808 Route de Lennik, Brussels, Belgium

e-mail: sgoldman@ulb.ac.be and the poor correspondence of $A B$ distribution with the cognitive manifestations of AD. Since the distribution of neurofibrillary degeneration is better correlated with the typology of cognitive deficits, tau might be a better choice in the search for valid $A D$ biomarkers. The initial attraction for $A B$ PET tracers also declined after the deceiving results of clinical studies aiming at amyloid clearance from AD patients' brain. These results have questioned the simplistic reasoning that equates disappearance of a single pathological feature with therapeutic benefit [3]. New questions have, therefore, been formulated such as, for instance, whether available amyloid PET tracers inform us about amyloid species that are the actual toxic actors in the pathophysiological process. The plea for a refined development of AD biomarkers has fuelled the search for PET tracers directed to tau deposits. This new effort is further encouraged by very recent and preliminary evaluations of therapeutic monoclonal antibodies that might reduce tau-related neurodegeneration [4]. Indeed, such new anti-AD strategies will require tools to assess the evolution of tau deposits in vivo. Such new tau tracers have been recently described [5, 6]. Data obtained with the $\left[{ }^{11} \mathrm{C}\right]$ PBB3 PET tracer are particularly promising since the uptake distribution in $\mathrm{AD}$ patients and in a patient with a corticobasal syndrome - a classic non-AD tauopathy - paralleled the distribution of tau deposits in these disorders.

The new category of tracers targeting large concentrations of amorphous protidic deposits raise validation issues that deserve some attention. Indeed, the early PET tracers belonged to two categories that cannot accommodate the new "protein load" tracers such as the $A ß$ and tau tracers. They were derived either from substrates of enzymes - e.g. hexokinase for FDG, dopa-decarboxylase for $\left[{ }^{18} \mathrm{~F}\right]$ fluorodopa - or from ligands of receptors and uptake sites present on the cell membrane. To validate those classic tracers, some rules were defined in the 1970s-80s, in accordance with those applied for in vitro enzymatic and binding experiments 
[7]. In particular, for in vivo binding experiments, competition and displacement challenges were crucial to differentiate specific from non-specific binding. In the neurotransmission machinery, specific binding sites are present at low concentrations; therefore, they are saturable and binding of the tracer in the presence of high concentrations of the ligand identifies the so-called non-saturable non-specific binding. Obviously, validation procedures based on image comparison in the presence of low versus high concentrations of ligands cannot be applied to "protein load" tracers that bind with high affinity to large amounts of brain deposits. Also, the binding of these tracers is not constrained by the high selectivity characterizing the binding of neurotransmitters to their respective receptors. The interaction of the new tracers with their targets is not based on subtle biochemical correspondences mimicking the complementarity between the natural ligand and the receptor; it relies on the chemical affinity of a "dye" with an organic molecule presenting some repetitive structural features. As a consequence, rules have changed in this new field; lack of selectivity does not disqualify a tracer. Binding of a brain $A B$ tracer on a totally different kind of protein in cardiac amyloidosis is not questioning its value as a biomarker of a selective pathophysiological feature; on the contrary, it is actually presented as another opportunity of use [8]. So, if competition with specific ligands and selectivity do not validate these "protein load" tracers, how do we ascertain that the signal they produce is representative of the pathological process scrutinized? Most of these tracers are radioactive derivatives of chemical compounds with high affinity for the target protein in vitro, even on fixed post-mortem material. So, the binding of these radioactive molecules is preliminarily assessed in vitro and this is a necessary step on the way to valid candidates [9]. Yet, success in vitro does not preclude in vivo binding on non-specific sites. Translation of in vitro binding assays into in vivo reality is further complicated by the compartmentalization of the target proteins in the patients' brain. This issue is particularly relevant to the tau protein, which primarily accumulates as intracellular neurofibrillary tangles. The same type of questioning holds true for tracer validation through in vivo imaging of transgenic animals overexpressing the protein of interest. Indeed, in terms of molecular form and conformation, tissue concentration and localization, the target protein in these animals might differ from deposits maturing in the brain of $\mathrm{AD}$ patients over several decades. Mathematical modelling used to analyse dynamic PET data in patients might help characterize the binding, but it is based on various assumptions that require experimental supporting data, and the dynamics of tracer accumulation is dependant on factors that are not easily controlled in the clinics $[10,11]$. To validate a tracer, can we then rely on the differential regional uptake distribution in healthy subjects' and patients' brains? This pertinent criterion is, for instance, highlighted in the recent work of Maruyama et al. on a new tau protein tracer [5]. It is actually used as a positive hint to encourage the community to adopt new tracers, but it is neglected when it is violated. Indeed, discordance between the distribution of $A \beta$ tracers with the known distribution of the deposits - and the neuronal dysfunction assessed with PETFDG - has not been considered to be challenging their potential clinical value. At the end of the day, the community essentially relies on purely clinical considerations to support the use of "protein load" tracers: when a tracer accumulates significantly more in the brains of individuals with a higher probability of being affected by a disease, this tracer is considered for further use as a biomarker of this disease.

The paper by Villemagne et al. [1] illustrates the difficulty in dealing with validation issues when it comes to tracers that pertain to protein load detection. Differences in the images produced in healthy subjects and in patients with $\mathrm{AD}$ constitute the main justification for presenting ${ }^{18} \mathrm{~F}$-THK523 as a selective tracer of tau in AD. The very high uptake of the tracer in the white matter leads the authors to discard the tracer for practical reasons: the simple visual inspection of the images would be impaired by the presence of a high background close to the cortical sites where the signal of interest should be evaluated. This reason is not valid to discard the tracer: ${ }^{15} \mathrm{O}$ labelled water remains one of the most accurate tracers for quantitative assessment of the myocardial blood flow, despite the strong signal this tracer produces in the cardiac cavities. The actual reason for discarding the ${ }^{18} \mathrm{~F}$-THK523 tracer is different. Since it produces a high non-specific signal in the white matter, the selective cortical signal it is supposed to produce ends up impossible to interpret unequivocally as such. To reach that conclusion, we need to consider the biological and methodological problems raised by tracers with high non-specific binding. By definition, non-specific binding is related to the retention of the tracer due to interactions with unknown cellular or extracellular molecules. So, until it is proven that the binding in the white matter is restricted to a component absent in the grey matter, the question remains open of a partial non-specific uptake of the same nature within the grey matter. Such a non-specific binding in the grey matter would be suspected, for instance, if white matter binding were related to glial structures. Until this question is answered, the cortical binding cannot be viewed as selectively related to the presence of the target. There is another reason to doubt the selectivity of the cortical signal produced by ${ }^{18} \mathrm{~F}$-THK523. To deal with the possible influence of the massive non-specific signal close to the cortex, the authors applied a partial volume correction (PVC) combined with an "atrophy correction" thanks to a three-compartment model run on a commercial software. The authors do not bring indices that this correction is validated in this particular situation of predominant uptake in the white matter combined with cortical atrophy. If we follow the succession of quotes on the PVC method applied, we end up with three references in an article of the group [12]. 
These references are essentially dealing with corrections applied to data with high uptake in the grey matter, a situation totally different from the present one - the most recent reference deals with a method developed for FDG data ten years ago [13]. It is noteworthy that all methods at the source of the one applied by Villemagne et al. consider white matter as a "low activity" region, and the phantom tests have been conducted accordingly (white matter/grey matter count values $=1 / 4$ ). So, obviously, the method applied is not validated for the management of ${ }^{18} \mathrm{~F}$-THK523 PET images where white matter values are twice the values in the grey matter of healthy controls. The data presented would deserve the application of new PVC methods, based on modern developments in the field [14]. This view is substantiated by the close examination of the images presented in the article by Villemagne et al. On the PV-corrected images, the uptake appears maximal at the very superficial aspect of the cortex (this is most obvious in healthy controls, on the transverse slice) and at the border of the sylvian scissure, raising questions about the PVC method. This observation might actually impact the interpretation of the results since the superficial signal in the "insula", interpreted as supporting the selectivity of the tracer, might well be related to variable sylvian scissure volumes, not adequately managed by the correction for partial volume effects.

In conclusion, we are witnessing the emergence of radiotracers that deviate from the classic precursors that gave birth to experimental and clinical PET imaging. Tracers of biochemical deposits accumulated in diseased organs are one type of new PET agent, but other newcomers are already there, such as antibody-like molecules, and peptidic and hybrid molecules labelled with new positron emitters. Thanks to the precise mastering of a theoretical background leading to a strict methodology, PET is regarded as the ultimate quantitative molecular imaging tool. To ensure the future of this technology, there is a need for an urgent renewal of its theoretical and methodological frame, in order to accommodate new classes of innovative molecules that require a revised approach for accurate image handling and analysis.

Acknowledgments Serge Goldman is supported by research grants from the "Fonds de la Recherche Scientifique" (FRS-FNRS, Belgium) and by the Fonds Erasme and the Fondation ULB.

Disclosure: The author reports no disclosure.

\section{References}

1. Villemagne VL et al. In vivo evaluation of a novel tau imaging tracer for Alzheimer's disease. Eur J Nucl Med Mol Imaging. 2014. doi:10. 1007/s00259-013-2681-7.

2. Klunk WE, Engler H, Nordberg A, Wang Y, Blomqvist G, Holt DP, et al. Imaging brain amyloid in Alzheimer's disease with Pittsburgh Compound-B. Ann Neurol. 2004;55:306-19. doi:10.1002/ana. 20009.

3. Becker RE, Greig NH, Giacobini E, Schneider LS, Ferrucci L. A new roadmap for drug development for Alzheimer's disease. Nat Rev Drug Discov. 2013;13:156. doi:10.1038/nrd3842-c2.

4. Yanamandra K, Kfoury N, Jiang H, Mahan TE, Ma S, Maloney SE, et al. Anti-tau antibodies that block tau aggregate seeding in vitro markedly decrease pathology and improve cognition in vivo. Neuron. 2013;80:402-14. doi:10.1016/j.neuron.2013.07.046.

5. Maruyama M, Shimada H, Suhara T, Shinotoh H, Ji B, Maeda J, et al. Imaging of tau pathology in a tauopathy mouse model and in Alzheimer patients compared to normal controls. Neuron. 2013;79: 1094-108. doi:10.1016/j.neuron.2013.07.037.

6. Okamura N, Furumoto S, Harada R, Tago T, Yoshikawa T, FoderoTavoletti M, et al. Novel $18 \mathrm{~F}$-labeled arylquinoline derivatives for noninvasive imaging of tau pathology in Alzheimer disease. J Nucl Med. 2013;54:1420-7. doi:10.2967/jnumed.112.117341.

7. Mintun MA, Raichle ME, Kilbourn MR, Wooten GF, Welch MJ. A quantitative model for the in vivo assessment of drug binding sites with positron emission tomography. Ann Neurol. 1984;15:217-27. doi:10.1002/ana.410150302.

8. Antoni G, Lubberink M, Estrada S, Axelsson J, Carlson K, Lindsjo L, et al. In vivo visualization of amyloid deposits in the heart with $11 \mathrm{C}$ PIB and PET. J Nucl Med. 2012;54:213-20. doi:10.2967/jnumed. 111.102053.

9. Klunk WE, Engler H, Nordberg A, Bacskai BJ, Wang Y, Price JC, et al. Imaging the pathology of Alzheimer's disease: amyloid-imaging with positron emission tomography. Neuroimaging Clin N Am. 2003;13:781-9. ix.

10. Price JC, Klunk WE, Lopresti BJ, Lu X, Hoge JA, Ziolko SK, et al. Kinetic modeling of amyloid binding in humans using PET imaging and Pittsburgh Compound-B. J Cereb Blood Flow Metab. 2005;25: 1528-47. doi:10.1038/sj.jcbfm.9600146.

11. Rodell A, Aanerud J, Braendgaard H, Gjedde A. Washout allometric reference method (WARM) for parametric analysis of [(11)C]PIB in human brains. Front Aging Neurosci. 2013;5:45. doi:10.3389/fnagi. 2013.00045 .

12. Bourgeat P, Chetelat G, Villemagne VL, Fripp J, Raniga P, Pike K, et al. Beta-amyloid burden in the temporal neocortex is related to hippocampal atrophy in elderly subjects without dementia. Neurology. 2010;74:121-7. doi:10.1212/WNL.0b013e3181c918b5.

13. Quarantelli M, Berkouk K, Prinster A, Landeau B, Svarer C, Balkay L, et al. Integrated software for the analysis of brain PET/SPECT studies with partial-volume-effect correction. J Nucl Med. 2004;45:192-201.

14. Erlandsson K, Buvat I, Pretorius PH, Thomas BA, Hutton BF. A review of partial volume correction techniques for emission tomography and their applications in neurology, cardiology and oncology. Phys Med Biol. 2012;57:R119-59. doi:10.1088/0031-9155/57/21/R119. 